\title{
ORIGINAL
}

\section{ESTIMACIÓN DE LA PREVALENCIA DE HIPOTIROIDISMO SEGÚN DIFERENTES MÉTODOS: DOSIS DIARIA DEFINIDA, DOSIS DIARIA PRESCRITA Y REGISTRO DE PACIENTES EN TRATAMIENTO}

\author{
José Escribano-Serrano (1), Carolina Paya-Giner (2), María Isabel Méndez Esteban (2), Manuela \\ Márquez-Ferrando (3), Antonio Zarallo-Pérez (4) y Alfredo Michán-Doña (5).
}

(1) Unidad de Gestión Clínica San Roque. Área de Gestión Sanitaria Campo de Gibraltar. Cádiz.

(2) Unidad de Gestión ClínicaFarmacia. Área de Gestión Sanitaria Campo de Gibraltar. Cádiz.

(3) Unidad de Gestión Clínica Farmacia. Distrito de Atención Primaria Bahía de Cádiz-La Janda. Cádiz.

(4) Unidad de Gestión Clínica Dr. Cayetano Roldán. Distrito de Atención Primaria Bahía de Cádiz-La Janda. Cádiz.

(5) UGC Medicina Interna-Dermatología. Área de Gestión Sanitaria Norte de Cádiz. Hospital de Jerez. Departamento de Medicina. Facultad de Medicina. Cádiz.

No existen conflictos de intereses.

\section{RESUMEN}

Fundamentos: El hipotiroidismo (HT) es una patología frecuente en la práctica clínica diaria. No existen datos recientes sobre su prevalencia en España. El objetivo de este trabajo fue conocer su prevalencia en la provincia de Cádiz.

Métodos: Se estudió el consumo del grupo terapéutico H03AA realizado en receta oficial por las unidades de Atención Primaria (AP) de Cádiz, durante el año 2012. La prevalencia se estimó mediante tres métodos: la Dosis Diaria Definida (DDD), la Dosis Diaria Prescrita (DDP) y el Registro Pacientes en Tratamiento (RPT). Los resultados se presentaron según sexo y edad con intervalos de confianza del $95 \%$.

Resultados: La prevalencia de HT durante 2012 en población mayor de 15 años de la provincia fue del 1,36\% (IC95: 1,33\%-1,38\%) mediante DDD, del 2,60\% (IC95: 2,54\%-2,66\%) mediante DDP y del $3,10 \%$ (IC95: 2,85\%-2,93\%) mediante RPT. La mediana de edad fue de 56 años (rango intercuartílico 43 a 68 años). La prevalencia (RPT) por sexo fue de $0,75 \%$ en hombres y $5,36 \%$ en mujeres [OR 7,26 (IC95: 7,02-7,52)], siendo del $9,35 \%$ la correspondiente a mujeres de 51 a 70 años.

Conclusiones: El método de estimación por RPTdemuestra ser una herramienta más válida que el método DDD para evaluar la prevalencia de HT. El HT muestra, en nuestra provincia una prevalencia elevada con mayor afectación del sexo femenino.

Palabras clave: Hipotiroidismo. Epidemiología. Prevalencia. Uso terapéutico de Levotiroxina.

\footnotetext{
Correspondencia

José Escribano-Serrano.

Avenida Castilla, 2

11360 San Roque

Cádiz

jescribanos@semergen.es
}

\section{ABSTRACT \\ Different Methods Used to Estimate the Prevalence of Hypothyroidism, Cadiz, Spain}

Background: Hypothyroidism (HT) is a common condition in clinical practice. There is a paucity of recent data on its prevalence in Spain, suggesting the need for an updated estimate and therefore the aim of this study was to determine its prevalence in the province of Cádiz.

Methods:We obtained data relative to medical prescriptions for group H03AA drugs issued in primary care centers attached to the Andalusian Health Service in the province of Cadiz in 2012. Prevalence was estimated on the basis of the defined daily dose (DDD), the prescribed daily dose (DDP) and treated patient records (TPR). We present the estimated prevalence and odds ratio by gender, with a confidence interval of $95 \%$.

Results: Prevalence of HT in 2012 in the population aged 15 years and over in the province of Cadiz was 1.36\% (CI95: 1.33\%-1.38\%) based on DDD; $2.60 \%$ (CI95: $2.54 \%-2.66 \%$ ) based on DDP; and $3.10 \%$ (CI95: $2.85 \%-2.93 \%$ ) based on TPR. Median age was 56 years (IQR: 43 to 68 ). Prevalence (TPR) by gender was $0.75 \%$ in men and $5.36 \%$ in women [OR 7.26 (CI95: 7.02-7.52)]. The greatest prevalence, $9.35 \%$, was found in the group of women aged 51 to 70 years.

Conclusions: Prevalence of HT in Cadiz is high, mainly affects women. Current prescribing systems allowed us to obtain TPRs, which have been shown to be a more valuable tool than DDDs for estimating HT prevalence.

Keyword: Hypothyroidism. Epidemiology. Prevalence. Levothyroxine therapeutic use. 


\section{INTRODUCIÓN}

El hipotiroidismo (HT) es la condición patológica más común ligada a un déficit hormonal. A pesar de ello y de su relación con distintos factores de riesgo vascular o del aumento de mortalidad ${ }^{1}$, los datos sobre su prevalencia son escasos.

Hace más de una década se publicó el que puede considerarse el último dato en población general de nuestro país ${ }^{2}$. Se estimó la prevalencia de HT en Lleida por distintos métodos: mediante el consumo en DDD de Levotiroxina (LT), que resultó del 0,54\%, y mediante registro de pacientes tratados, que fue el $0,84 \%$.

Se destacaron tres características propias del HT: (a) el uso habitual de dosis inferiores a $150 \mathrm{mcg}$ de LT (estipulada como DDD) que explicaría la diferencia de prevalencias; (b) una mayor afectación del sexo femenino; (c) baja prevalencia en la población menor de 15 años.

Previamente se habían publicado otros resultados estimados también mediante la $\mathrm{DDD}^{3,4}$. Morant y cols ${ }^{4}$ describieron la única referencia a Cádiz. La prevalencia del $0,24 \%$ que mostraba entonces junto al $0,17 \%$ de Jaén eran las menores tasas de prevalencia en España.

Los estudios de campo han sido escasos y dispares, bien por diferencias de población estudiada, bien por discrepancias de los puntos de corte utilizados para el diagnóstico de HT o fundamentalmente por su laboriosidad. A los largo de estos años se han ido publicando diversos trabajos ${ }^{5-14}$ en referencia a la prevalencia de HT, pero realizados bien en territorios pequeños bien en grupos poblacionales muy específicos.

Los datos existentes sobre poblaciones extranjeras son igualmente escasos. La epidemiologia del HT descrita en la revisión de Vanderpump ${ }^{15}$ en las Guías de la Sociedad
Americana del Tiroides ${ }^{16}$ o en el reciente meta-análisis de Garmendia ${ }^{17}$ se basa en estudios clásicos del siglo XX.

Por otra parte, en la práctica clínica actual dentro del Sistema Público de Salud (SPS) se dispone de medios informáticos con capacidad de almacenar información en la historia clínica sobre las prescripciones ${ }^{18}$, lo que permite un análisis pormenorizado del consumo de medicamentos ${ }^{19}$.

Los objetivos de este estudio fueron estimar la prevalencia de HT en la provincia de Cádiz durante el año 2012 y conocer su distribución por sexo y grupos de edad.

\section{MATERIAL Y MÉTODOS}

Población de estudio. Se realizó un estudio observacional que analizó el consumo del grupo terapéutico H03AA de la Clasificación Anatómica Terapéutica (H Hormonas, 03 Preparados tiroideos y AA Hormonas tiroideas) realizadas por los médicos de atención primaria (AP) de la provincia de Cádiz y financiadas con cargo al SPS.

Cádiz es la provincia más meridional de la península ibérica y es la octava provincia española por población, con aproximadamente 1.245.000 habitantes. El SPS organiza la AP de la provincia en tres distritos o áreas (Bahía de Cádiz-La Janda, Norte de Cádiz y Campo de Gibraltar), las cuales cuentan con 50 Unidades de Gestión Clínica de AP (UGC-AP).

Se utilizaron los datos de la población con derecho a asistencia sanitaria por el SPS en lugar de los de la población censada(aproximadamente al 95\% de la población general) dado que el resto puede disponer de una financiación de medicamentos distinta. Los usuarios se identificaron por el número único de la historia de salud de Andalucía (NUHSA), obtenidos de la base de datos de usuarios (BDU) del SPS de Andalucía. La población registrada al ini- 
cio de 2012 era de 1.185.788 NUHSA, siendo el $51 \%$ mujeres.

Parámetros analizados. En España solo se comercializa un principio activo del grupo H03AA, la levotiroxina sódica (LT), con tres presentaciones disponibles en el momento del estudio: Dexnon ${ }^{\circledR}$ (dosis de $100 \mathrm{mcg}$ ), Eutirox ${ }^{\circledR}$ (de 25, 50, 75, 88, 100, $112,125,137,150,175$ у $200 \mathrm{mcg})$ у Levothroid® (de 50 y $100 \mathrm{mcg})$.

Los datos de consumo se obtuvieron de la base de datos de prescripciones del Servicio Andaluz de Salud (MicroStrategy Farma ${ }^{\circledR}$ ). Para analizar los datos, los estudios mostraron diferencias significativas si se calculaba mediante la DDD o el registro de pacientes (1) e incluso puede utilizarse la DDP (19). Por ello se decidió estimar la prevalencia de HT de tres formas distintas:

1. La prevalencia mediante la $\mathrm{DDD}^{18}$, definida como la dosis media diaria habitual de un medicamento cuando éste se utiliza para su indicación principal y en sujetos adultos no gestantes. La Organización Mundial de la Salud (OMS) específica que para LT es de 150 microgramos.

2. Prevalencia mediante $\mathrm{DDP}^{18}$, definida como como la dosis media diaria verdadera que toma cada paciente cuando usa un fármaco en su indicación principal ${ }^{19}$. Los resultados del consumo anual se expresan en número de DDD, por lo que deben ser corregidos de acuerdo a la dosis consumida por cada paciente, multiplicando la cantidad consumida en DDD por una constante $\mathrm{k}$ (resultado de dividir la dosis definida, 150 mcg, por el tipo de dosis prescrita al sujeto). Por ejemplo, un paciente que tome $75 \mathrm{mcg}$ al día consumiría 0,5 DDD, que se convertirían en 1 DDP $[0,5$ por 2 (resultado de 150/75)].

3. La prevalencia por registro de $\mathrm{NUH}-$ SA en tratamiento (RPT): los sistemas informáticos permiten obtener datos de con- sumo con un registro individualizado de cada paciente en tratamiento, identificados por su NUHSA.

Los registros de consumo detallan el tipo de dosis consumida, el sexo y la edad del sujeto así como los centros de procedencia, lo que permite distribuir los resultados por sexo, grupos etarios (menores de 15 años, de 15 a 30 , de 31 a 50 , de 51 a 70 y mayores de 71 años), UGC-AP y Comarca.

Estrategia de análisis. En primer lugar se estimó la prevalencia en la población general mediante la DDD para comparar con los antiguos estudios de prevalencia ${ }^{2,4}$. Se confirmaron las tres características destacadas por Serna y cols ${ }^{2}$ : importante asimetría entre sexos, baja prevalencia en los menores de 15 años y uso habitual de una dosis por debajo de la DDD en la mayoría de los pacientes.

A continuación se estimó la prevalencia mediante DDD, DDP y RPT para las personas mayores de 15 años, estratificando por sexo y grupo de edad. Se expresó en porcentaje acompañado de sus correspondientes intervalos de confianza al 95\%. Las variables continuas, dosis y edad, se enunciaron con la mediana (M) y el rango intercuartílico (RIC). El estudio fue aprobado por el Comité de Ética e Investigación Clínica del Hospital Universitario de Puerto Real, del Área Bahía de Cádiz- La Janda y del Área Sanitaria Campo de Gibraltar.

\section{RESULTADOS}

Durante el año 2012, 33.859 sujetos (NUHSA) consumieron 5.370.358 DDD de LT, convertidas en 10.343.348 DDP sobre una población 1.185 .788 personas. Se estimó una prevalencia para la población general por DDD del 1,24\% (IC95: 1,22\%1,27\%), por DDP del 2,39\% (IC95: 2,36\%$2,43 \%$ ), y por RPT del 2,86\% (IC95: $2,82 \%$ 2,90\%) (figura 1). 
Figura 1

Prevalencia de HT en la provincia de Cádiz para la población general estimada por los tres parámetros: Dosis Diaria Definida (DDD), Dosis Diaria Prescrita (DDP) y Registro de Pacientes Tratados (RPT)

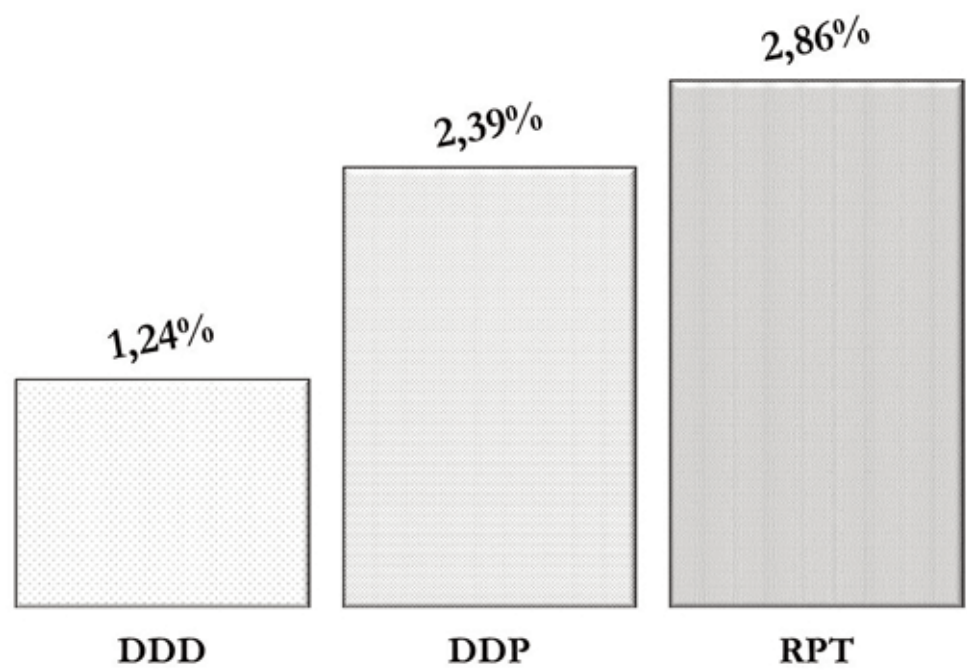

Figura 2

Medianas de las dosis prescritas de LT (mcg) estratificadas por sexo y grupos de edad. Hombre (Circulo vacío) Mujer (Cuadrado lleno). Barras representan percentil 25/75 y asterisco significa presencia de diferencias significativas entre $\operatorname{sex} 0 s(p<0,01)$

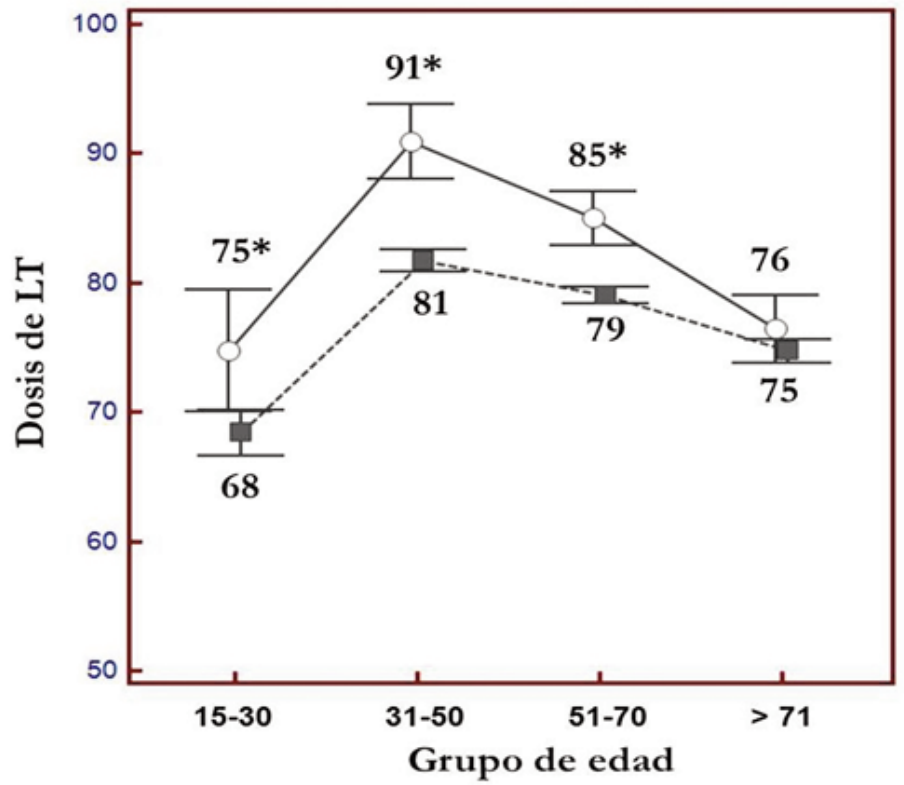


Figura 3

Prevalencia de HT en la provincia de Cádiz para población mayor de 15 años, estimada por los tres parámetros: Dosis Diaria Definida (DDD), Dosis Diaria Prescrita (DDP) y Registro de Pacientes Tratados (RPT)

\section{Población mayor de 15 años}

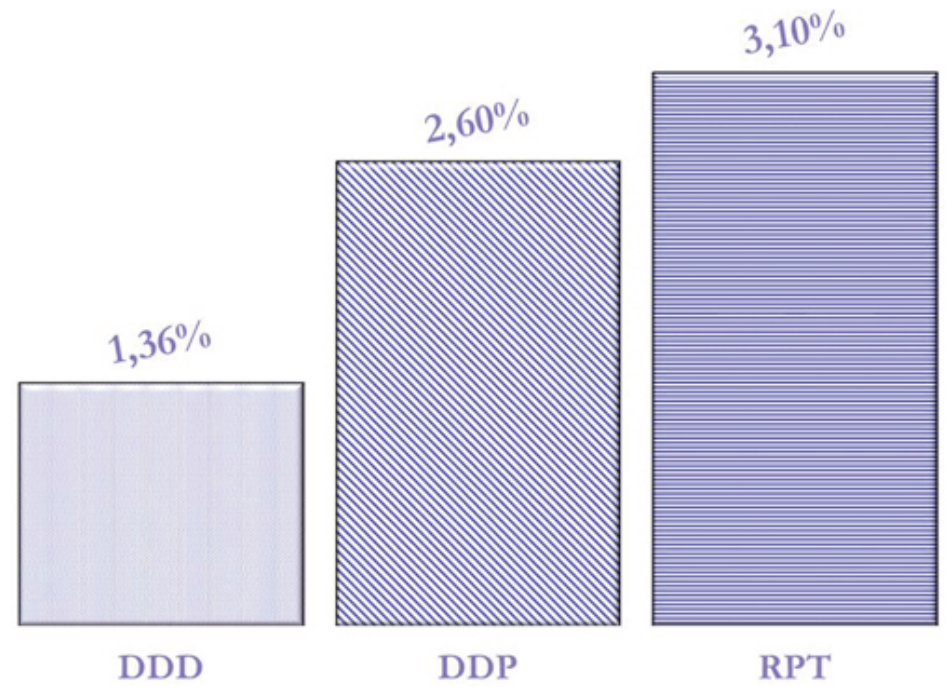

Tabla 1

Prevalencias de HT en la provincia de Cádiz para población mayor de 15 años, estimadas por los tres parámetros: Dosis Diaria Definida (DDD), Dosis Diaria Prescrita (DDP) y Registro de Pacientes Tratados (RPT) estratificada por sexo con sus correspondientes intervalos al $95 \%$

\begin{tabular}{|l|c|c|c|c|c|}
\hline \multicolumn{1}{|c|}{ Método } & $\mathrm{n}$ & $\begin{array}{c}\text { Prevalencia } \\
(\mathrm{IC95} \%)\end{array}$ & $\begin{array}{c}\text { Prevalencia } \\
\text { mujeres } \\
(\mathrm{IC95} \%)\end{array}$ & $\begin{array}{c}\text { Prevalencia } \\
\text { hombres } \\
(\text { IC95\%) }\end{array}$ & $\begin{array}{c}\text { OR } \\
\text { sexos }\end{array}$ \\
\hline DDD & $\begin{array}{c}5.341 .222 \\
\text { DDD }\end{array}$ & $\begin{array}{c}1,36 \% \\
(1,33 \text { a } 1,38)\end{array}$ & $\begin{array}{c}2,34 \% \\
(2,31 \text { a } 2,37)\end{array}$ & $\begin{array}{c}0,34 \% \\
(0,32 \text { a } 0,36)\end{array}$ & $\begin{array}{c}6,94 \\
(6,82 \text { a } 7,82)\end{array}$ \\
\hline DDP & $\begin{array}{c}10.255 .858 \\
\text { DDP }\end{array}$ & $\begin{array}{c}2,60 \% \\
(2,56 \text { a } 2,64)\end{array}$ & $\begin{array}{c}4,52 \% \\
(4,48 \text { a } 4,56)\end{array}$ & $\begin{array}{c}0,60 \% \\
(0,57 \text { a } 0,62)\end{array}$ & $\begin{array}{c}7,85 \\
(7,82 \text { a } 7,88)\end{array}$ \\
\hline RPT & $\begin{array}{c}33.451 \\
\text { Pacientes }\end{array}$ & $\begin{array}{c}3,10 \% \\
(2,85 \text { a } 2,93)\end{array}$ & $\begin{array}{c}5,36 \% \\
(5,31 \text { a } 5,41)\end{array}$ & $\begin{array}{c}0,75 \% \\
(0,70 \text { a } 0,79)\end{array}$ & $\begin{array}{c}7,26 \\
(7,02 \text { a } 7,52)\end{array}$ \\
\hline
\end{tabular}




\section{Figura 4}

Prevalencia de HT en la provincia de Cádiz para la población mayor de 15 años, estratificada por sexo y grupos de edad, estimada únicamente por el Registro de Pacientes

\section{Tratados (RPT)}

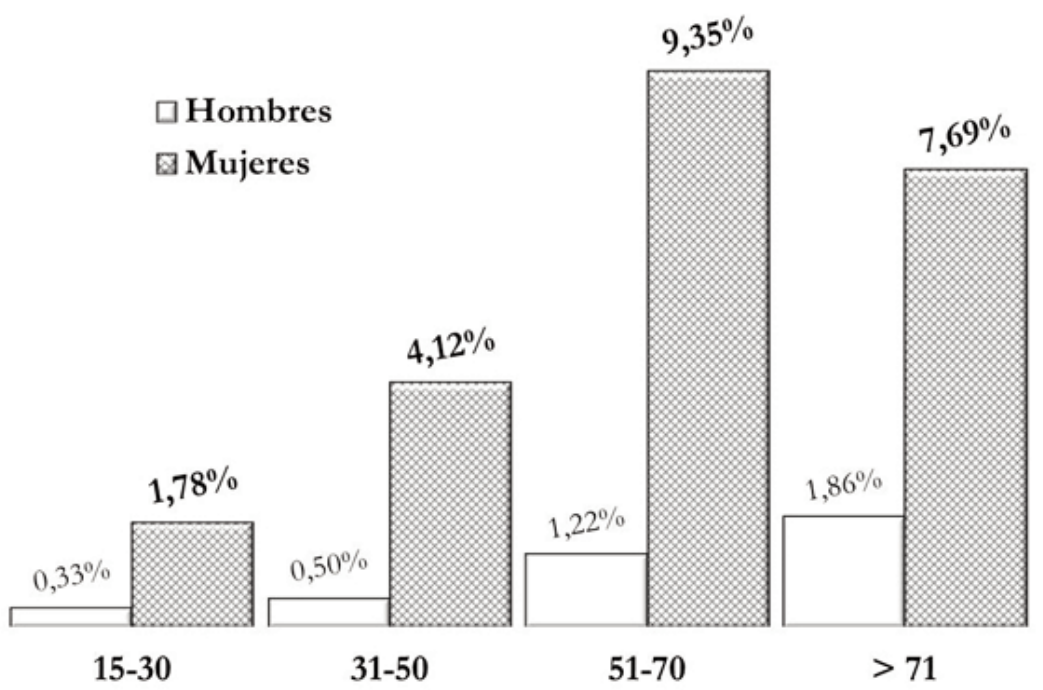

La mediana de edad de las personas que consumían LT fue de 56 años (RIC: 43-68), similar en ambos sexos. De dicha población se encontraba por encima de los 50 años el $75 \%$ de los hombres y el $55 \%$ de las mujeres.

El 95\% de los pacientes consumía LT en dosis inferiores a la DDD de $150 \mathrm{mcg}$. La mediana de dicha dosis fue de $75 \mathrm{mcg}$ (RIC 50-100), en hombres de 83 mcg (RIC 50111) y en mujeres de 75 mcg (RIC 50-100) $(\mathrm{p}<0,01)$. Las medianas de las dosis consumidas por cada sexo y grupo de edad se reflejan en la figura 2.

Los resultados de la prevalencia de HT en la población adulta se estimaron por los métodos ya citados (figura 3 ). Destacó la prevalencia por RPT del 3,1\% (IC95: $1,22 \%-1,27 \%)$, con una razón de sexos de 7:1 para la mujer [OR 7,17 (IC95: 6,937,41)] (tabla 1).

En la figura 4 se detallan las prevalencias estratificadas por sexo y grupo etario, destacando los niveles que se alcanzan en el grupo de mujeres entre 51 y 70 años, que llegan a alcanzar el 9,35\% (IC95: 9,32\%-9,38\%). En el sexo femenino la prevalencia aumenta en relación al grupo de edad, así el grupo de 30-50 supera al anterior en más del doble [OR 2,33 (IC95: 2,22-2,44) y por encima de los 50 años casi se triplicó [OR 2,65 (IC95: 2,58-2,71)].

\section{DISCUSIÓN}

Nuestro estudio pone de manifiesto la importancia del método seleccionado para la estimación de la prevalencia de HT y de la población sobre la que se estima, dado que existen diferencias entre los distintos métodos utilizados para cada población. Consideramos que con el RPT en la población mayor de 15 años se obtienen los mejores resultados y es un parámetro que puede obtenerse fehacientemente con los actuales registros informáticos. De hecho, basándonos en el RPT podemos afirmar que la 
prevalencia de HT en la provincia de Cádiz para el año 2012 alcanzó el 3,1\% de la población mayor de 15 años, que el HT es una patología que afecta fundamentalmente al sexo femenino $(5,36 \%)$ y que el uso de la DDD para medir la prevalencia se ve ampliamente superado por el RPT e incluso por la DDP, parámetros que los registros actuales permiten medir.

Dada la ausencia de referencias recientes sobre la prevalencia de HT en nuestro entorno es difícil establecer una comparación. Más aún si se tiene en cuenta que los últimos estudios realizados en poblaciones grandes utilizaron la DDD como método de estimación ${ }^{2-4}$.

Sin embargo, a través de datos obtenidos de forma indirecta, podemos realizar una comparación aproximada. Orueta y cols $^{13}$ revisaron la prevalencia de nueve condiciones crónicas en el País Vasco durante 2008, manejando diversas bases de datos. Aportan una prevalencia de HT del $2,71 \%$ cuando utilizan solo el registro de prescripción de medicamentos y aumenta al 3,21\% cuando combinan varias.

El estudio Di@bet.es realizado durante 2010 en población española mayor de 18 años, cuyo objetivo era conocer la prevalencia de diabetes, investigó la medicación de los participantes. Se analizaron ocho grupos de fármacos, entre ellos la LT, y se encontró que el $4,15 \%$ de la población estudiada consumía $\mathrm{LT}^{14}$.

En Europa, el estudio realizado por Virta $\mathrm{y} \mathrm{col}^{20}$ en población general de Finlandia durante el año 2007, con una metodología similar a la del presente estudio, encontró una prevalencia del 3,58\% (IC95: 3,57\%-3,60\%). La consulta en línea de las bases de datos de consumo de fármacos en adultos durante el año 2012 nos aportan para Suecia unos porcentajes de población consumiendo LT del
$4,94 \%^{21}$, para Dinamarca del $3,30 \%{ }^{22}$ y para Noruega del $3,73 \%{ }^{23}$.

Teniendo en cuenta los datos previos ${ }^{4}$, en los que Cádiz se encontraba entre las provincias de menor prevalencia del país, y los distintos años de realización, nuestros resultados concuerdan con las publicaciones españolas $1^{13,14}$ o europeas ${ }^{20-23}$.

La mayor frecuencia de la prevalencia en mujeres es una constante presente en los estudios que la han analizado. Por ejemplo en el Diabet.es (OR 6,85) ${ }^{14}$, en Finlandia $(\mathrm{OR} 4,5)^{20}$, en Suecia (OR 4,9)21 o en Noruega (OR 4,5) ${ }^{23}$. Incluso, al examinar los datos de una forma más detallada, es muy distinta la prevalencia en la mujer para los distintos grupos etarios, aunque no se produce un aumento directamente relacionado con el aumento de la edad, como ocurría en otras publicaciones ${ }^{20}$. Ante cualquier aproximación al estudio del HT se debería dar una especial importancia al sexo y en la mujer a la edad

Concretando más, existen importantes diferencias de prevalencia en las mujeres según sean menores o mayores de 50 años, de forma similar a lo expuesto en el Diabet.es ${ }^{14}$ pero menor que la descrita en los países nórdicos ${ }^{21-23}$. Extrapolando los datos, se podría afirmar que una de cada diez mujeres por encima de esa edad se encuentra en tratamiento con LT. Incluso dentro del grupo de mujeres menores de 50 años existen diferencias a partir de los 30. Este dato puede resultar relevante y aportar conocimiento en la polémica actual sobre la indicación o no de un cribado obligatorio de la disfunción tiroidea en la mujer gestante ${ }^{24}$. De acuerdo con las prevalencias de este estudio, la edad de la mujer gestante debería ser un factor determinante y en las mayores de 30 años la indicación de cribado debería estar clara.

Se podría esperar que la estimación de la 
prevalencia según el consumo de fármacos fuera mayor que la obtenida por registro de pacientes. Sin embargo dos factores van en contra de ello. Por una parte la posible falta de adherencia al tratamiento en una enfermedad crónica y poco sintomática y, por otra, el muy frecuente uso de dosis inferiores a la estipulada como DDD a nivel internacional.

Usar la DDD para estimar la prevalencia de HT debería descartarse. Este estudio corrobora en población general (RPT 2,86\% vs DDD $1,24 \%$ ) lo que otros ya habían planteado: Sendra y cols (RPT $0.84 \%$ vs DDD $0.54 \%)^{2}$ o Virta y cols. $(4,15 \% \text { vs } 2,39 \%)^{20}$ y lo reafirma para la población mayor de 15 años. La mayoría de los sujetos están siendo tratados con LT a una dosis inferior a la DDD definida. La dosis habitualmente utilizada está alrededor de los $90 \mathrm{mcg}$, similar a la usada en Finlan$\mathrm{dia}^{20}$. Esta dosis parece estar más relacionada con el sexo y el peso que con la edad del paciente ${ }^{25}$. Por ello, descartar la DDD parece lógico, ya que es un parámetro diseñado para realizar cálculos poblacionales cuando no se dispone de datos individuales ${ }^{18}$. Sin embargo, si los datos individualizados están disponibles, el RPT parece un parámetro más válido para estimar la prevalencia. Y en el caso de disponer únicamente de datos de consumo, estimar mediante la DDP sería un mejor planteamiento, aunque sus estimaciones pueden resultar algo inferiores a las obtenidas por RPT. La explicación estaría en la influencia de los tratamientos que no hayan completado todas las dosis asignadas al periodo de estudio sobre la DDP, bien por su comienzo durante ese periodo o por la falta de adherencia a la medicación.

Este estudio tiene las limitaciones propias de la metodología utilizada. La LT tiene otras indicaciones, aunque de menor entidad, como pueden ser el tratamiento combinado del hipertiroidismo, del cáncer tiroideo o la hipotiroxinemia gestacional. La prevalencia se estimó a partir de los pacientes que consumen LT con cargo al sistema público, por lo que sólo es posible conocer la prevalencia del HT diagnosticado y tratado. La LT es además un medicamento barato y de fácil adquisición, aunque no se ha podido medir el consumo privado. Tampoco es posible medir aquellas situaciones de HT oculto, de pautas anormales de tratamiento o las de inercia terapéutica. $\mathrm{Si}$ bien estas limitaciones pueden resultar frecuentes, generalmente darán lugar a una infraestimación de la prevalencia real, lo cual no resta importancia a los resultados de este estudio.

Otra dificultad, exclusiva del HT y más difícil de cuantificar, sería la provocada por el uso de fármacos en los pacientes con HT subclíni$\mathrm{co}^{26}$. La utilización de LT en los pacientes con niveles adecuados de tiroxina libre pero con niveles de TSH por debajo de $10 \mathrm{mU}$ queda, según las guías de práctica clínica, a criterio del médico prescriptor ${ }^{16,27}$. Por tanto, su influencia en la estimación de la prevalencia del hipotiroidismo siempre resultará imprecisa.

Por otra parte, las recomendaciones sobre la gestión del HT que realizan las guías de práctica clínica o las de los procesos asistencia$\operatorname{les}^{16,27}$ no reflejan la realidad de esta patología. La ausencia de datos actualizados de prevalencia puede estar en la raíz de esta cuestión. Son precisos más estudios en esta línea, pero en futuras revisiones de las recomendaciones, se debería tener en consideración este aumento de la prevalencia, reflejo de la realidad.

Podemos concluir afirmando que el HT tiene actualmente una alta prevalencia en nuestra provincia, siendo más frecuente en mujeres, quizás como consecuencia de la concienciación de los profesionales y de la mejora en la disponibilidad y calidad de las determinaciones diagnósticas.

La mayor aplicabilidad de este trabajo ha sido demostrar la utilidad de la estimación de prevalencias mediante el registro de pacientes, en este caso del HT, también útil para otras enfermedades crónicas. 


\section{AGRADECIMIENTOS}

Los autores quieren mostrar su agradecimiento al Dr. Juan Antonio Córdoba Doña (Delegación Territorial de Igualdad, Salud y Políticas Sociales, Cádiz, España) por sus valiosas aportaciones.

\section{BIBLIOGRAFÍA}

1. McQuade C, Skugor M, Brennan DM, Hoar B, Stevenson C, Hoogwerf BJ. Hypothyroidism and moderate subclinical hypothyroidism are associated with increased all-cause mortality independent of coronary heart disease risk factors: a PreCIS database study. Thyroid. 2011; 21(8):837-43.

2. Serna Arnáiz MC, Galván Santiago L, Gascó Eguiluz E, Manrique Manrique M, Foix Oña MM, Martín Gracia E. Estimación de la prevalencia de hipotiroidismo en Lleida a partir de la prescripción de hormonas tiroideas. Rev Esp Salud Pública. 2003; 77(3):405-10.

3. Díaz Madero A, López Ferreras A. Estimación de la prevalencia del hipotiroidismo en Castilla y León y su evolución desde 1992 al 2000 a través del consumo de hormonas tiroideas. Rev Esp Salud Pública. $2001 ; 75(4): 345-52$.

4. Morant Ginestar C, Criado-Alvarez JJ, García-Pina R, Pérez Garrido B. Estimación de la prevalencia de hipotiroidismo en España a partir del consumo de hormonas tiroideas (1996-1999). Rev Esp Salud Pública. 2001; 75(4):337-44.

5. Sempere Verdú E, Feliu Sagala M, Hernández Ruiz R, Ajenjo Navarro A. Prevalencia del hipotiroidismo tratado en la población adulta. Aten Primaria. 2005;35(3):163-4.

6. Díez JJ, Molina I, Ibars MT. Prevalence of thyroid dysfunction in adults over age 60 years from an urban community. Exp Clin Endocrinol Diabetes. 2003; 111(8):480-5.

7. Sender Palacios MJ, Vernet Vernet M, Pérez López S, Faro Colomés M, Rojas Blanc M, Pallisa Gabriel L. Enfermedad funcional tiroidea en la población de edad avanzada. Aten Primaria. 2004; 34(4):192-7.

8. Iglesias P, Muñoz A, Prado F, Guerrero MT, Macías MC, Ridruejo E, et al. Alterations in thyroid function tests in aged hospitalized patients: prevalence, aetiology and clinical outcome. Clin Endocrinol (Oxf). 2009; 70(6):961-7.
9. Iglesias P, Lázaro J, Velasco G, Díez JJ. Disfunción tiroidea en población laboral hospitalaria. Rev Clín Esp. 2010; 210(10):505-8.

10. Ballester LV, Candil SD, Reymunde TI, Escofet FS, Henzi FT, Jaramillo ST, et al. Assessment of dietary habits related to iodine intake and iodine concentration and thyroid dysfunction in a non-preselected population in Spain (the Thyrobus Project). Endocrinol Nutr. 2010; 57(9):407-13.

11. Lorenzo Gómez T, Cardelle Pérez F, De Las Heras Liñero E. The high prevalence of thyroid dysfunction in psychiatric inpatients. Rev Psiquiatr Salud Ment. 2010; 3(1):23-6.

12. Lucas A, Julián MT, Cantón A, Castell C, Casamitjana R, Martínez-Cáceres EM, et al. Undiagnosed thyroid dysfunction, thyroid antibodies, and iodine excretion in a Mediterranean population. Endocrine. 2010; 38(3):391-6.

13. Orueta JF, García-Álvarez A, García-Goñi M, Paolucci F, Nuño-Solinís R. Prevalence and costs of multimorbidity by deprivation levels in the basque country: a population based study using health administrative databases. PloS One. 2014; 9(2):e89787.

14. Rojo-Martínez G, Valdés S, Colomo N, Lucena MI, Gaztambide S, Gomis R, et al. Use of Drugs Related to the Treatment of Diabetes Mellitus and Other Cardiovascular Risk Factors in the Spanish Population. The Di@bet.es Study. Rev Esp Cardiol. 2013; 66(11):854-63.

15. Vanderpump MPJ. The epidemiology of thyroid disease. Br Med Bull. 2011; 99:39-51.

16. Garber JR, Cobin RH, Gharib H, Hennessey JV, Klein I, Mechanick JI, et al. Clinical practice guidelines for hypothyroidism in adults: cosponsored by the American Association of Clinical Endocrinologists and the American Thyroid Association. Thyroid 2012; 22(12):1200-35.

17. Garmendia Madariaga A, Santos Palacios S, Guillén-Grima F, Galofré JC. The Incidence and Prevalence of Thyroid Dysfunction in Europe: a Meta-analysis. J Clin Endocrinol Metab. 2014; 99(3):923-31.

18. Laporte JR CD. Métodos aplicados en estudios descriptivos de utilización de medicamentos. Principios de epidemiología del medicamento. Segunda Edición. Barcelona: Masson Salvat; 2007.

19. Capellà D Laporte JR. Principios de epidemiología del medicamento - Capítulo 4 [Internet]. [citado 9 de febrero de 2014]. Disponible en: http://www.icf.uab.es/pem/cap4.asp 
20. Virta LJ, Eskelinen SI. Prevalence of hypothyroidism in Finland, a nationwide prescription study. Eur J Clin Pharmacol. 2011; 67(1):73-7.

21. Statistikdatabas för läkemedel [Internet]. [Citado 3 de mayo de 2014]. Disponible en: http://www.socialstyrelsen.se/statistik/statistikdatabas/lakemedel

22. Statens Serum Institut - Statistikker. [Citado 3 de mayo de 2014]. Disponible en: http://www.medstat.dk/en

23. NorPD: Prevalence statistics. [Citado 3 de mayo de 2014]. Disponible en: http://www.norpd.no/Prevalens.aspx

24. Vila L, Velasco I, González S, Morales F, Sánchez $\mathrm{E}$, Torrejón S, et al. Controversies in endocrinology: On the need for universal thyroid screening in pregnant women. Eur J Endocrinol. 2014; 170(1):R17-30.

25. Devdhar M, Drooger R, Pehlivanova M, Singh G, Jonklaas J. Levothyroxine replacement doses are affected by gender and weight, but not age. Thyroid. $2011 ; 21(8): 821-7$.

26. Taylor PN, Iqbal A, Minassian C, Sayers A, Draman MS, Greenwood R, et al. Falling threshold for treatment of borderline elevated thyrotropin levels-balancing benefits and risks: evidence from a large community-based study. JAMA Intern Med. 2014; 174(1):32-9.

27. Sendón Perez AM (Coordinador). Proceso Asistencial Integrado Disfunción Tiroidea. Sevilla: Junta de Andalucía; 2003 [citado 1 de mayo de 2014]. Disponible en: http://www.juntadeandalucia.es/salud/sites/csalud/contenidos/Informacion_General/p_3_p_3 procesos_asistenciales_integrados/listado_procesos?perfil $=$ org 